\title{
Myocardial infarction with normal coronary arteries and factor XII deficiency
}

\author{
W J PENNY, B T COLVIN, N BROOKS \\ From London Chest Hospital, London; London Hospital, London; and Wythenshawe Hospital, Manchester
}

SUMMARY A 17 year old girl had a myocardial infarction. Three days later coronary angiography showed an occlusive thrombus in the circumflex and anterior descending arteries. The coronary arteries themselves appeared to be normal, and this was confirmed at repeat angiography three months later. Tests of haemostasis showed a deficiency of factor XII.

Most patients with acute myocardial infarction have atherosclerotic coronary disease, but subsequent coronary angiography sometimes does not show any evidence of chronic arterial obstruction. ${ }^{2}$ Spontaneous lysis of a thrombotic occlusion and resolution of spasm are two possible explanations for such observations, and both have been known to cause myocardial infarction as a complication of coronary angiography.

We recently showed an occlusive thrombus by coronary arteriography in a 17 year old girl three days after acute myocardial infarction; repeat angiography three months later showed normal coronary arteries. Tests of haemostasis confirmed a factor XII deficiency, a condition that has previously been associated with the occurrence of spontaneous intravascular thrombosis. ${ }^{3}$

\section{Case report}

A 17 year old girl was admitted to hospital having vomited and collapsed at home. On admission she was fully conscious but complained for the first time of severe pain in the centre of her chest that radiated through to her back. She had previously been well with no relevant inistory, and there was no family history of heart disease. She had smoked about 10 cigarettes daily for two years but was not taking the contraceptive pill.

Physical examination yielded normal results apart from a pulse rate of 120 beats/minute, and her blood pressure was $110 / 80 \mathrm{~mm} \mathrm{Hg}$. Serial electrocardiograms showed evolving changes in the ST segment, $T$ wave, and $Q$ wave typical of acute transmural

Requests for reprints to Dr W J Penny, London Chest Hospital, Bonner Road, London E2 9JX. inferolateral myocardial infarction. Serum aspartate transaminase activity rose to $166 \mathrm{IU} / 1$ (normal range 0-18 IU/1) and hydroxybutyrate dehydrogenase activity to $1108 \mathrm{IU} / 1$ (normal range 0-140 IU/1). Twenty four hours after admission she developed an apical third sound and crepitations at both lung bases, and her chest radiograph showed pulmonary oedema. These signs resolved after treatment with oral frusemide. Three days after admission she underwent coronary angiography and left ventriculography. These showed extensive lobulated filling defects in the left anterior descending and circumflex coronary arteries (Fig. 1). The left main and right coronary arteries were normal. The left ventriculogram showed extensive apical akinesia with mural thrombus. Treatment with warfarin was started. Her subsequent progress was uncomplicated, and the diuretic was stopped when she was discharged from hospital 10 days after admission.

The possibility that a venous thrombus may have embolised to the coronary circulation was explored by cross sectional echocardiography with contrast; there was no evidence of right to left shunting during a Valsalva manoeuvre. Three months later coronary arteriography was repeated. The coronary arteries appeared to be entirely normal (Fig. 2), and the clot previously seen in the apex of the left ventricle had disappeared. The anteroapical segment, however, remained akinetic. Treatment with warfarin was stopped, and she was subsequently given aspirin $300 \mathrm{mg}$ daily and dipyridamole $100 \mathrm{mg}$ thrice daily. A full blood count, measurement of erythrocyte sedimentation rate and electrolyte concentrations, and liver function tests yielded normal results. Tests for antinuclear factor were negative, and fasting lipid and cholesterol concentrations were normal. 

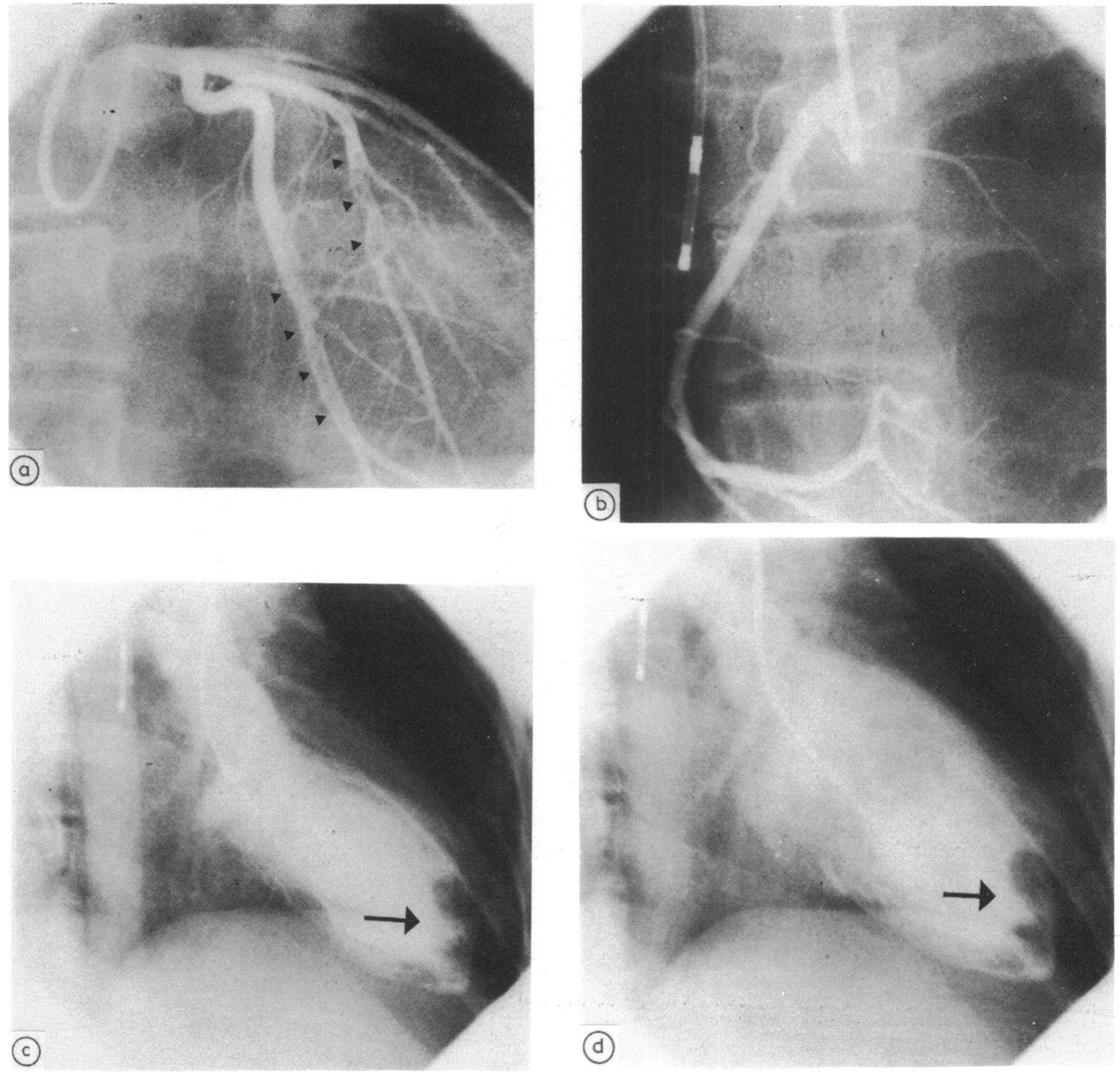

Fig. 1 Coronary arteriogram three days after onset of symptoms. (a) There were lobulated filling defects (arrowheads) in the left anterior descending and circumflex arteries. (b) The right coronary artery was normal. (c) and (d) The left ventriculogram in the right anterior oblique projection in systole (c) and diastole (d) showed extensive apical akinesia with mural thrombus (arrow).

TESTS OF HAEMOSTASIS

Blood was taken by clean venepuncture into $3.13 \%$ HEPES buffered sodium citrate at a dilution of one to 10. Plasma rich in platelets was prepared by centrifugation at $250 \mathrm{~g}$ for 10 minutes and plasma low in platelets by centrifugation at $2000 \mathrm{~g}$ for 20 minutes. The prothrombin time was measured with British Comparative Thromboplastin and the kaolin cephalin time with a commercial kit (Diagnostic Reagents Limited, Thame). The thrombin time reagent was a mixture of thrombin and calcium chloride adjusted to give a control time of 10 seconds.

Factor VIII concentration was measured by a method in two stages (Diagnostic Reagents Limited, Thame) and concentrations of factors IX, XI, and XII and prekallikrein were measured by one stage assays with known deficient plasmas as substrate. ${ }^{4} \mathrm{~A}$ test system for the assay of high molecular weight kininogen was not available. Antithrombin III and antiplasmin concentrations were assayed by amidoly- 

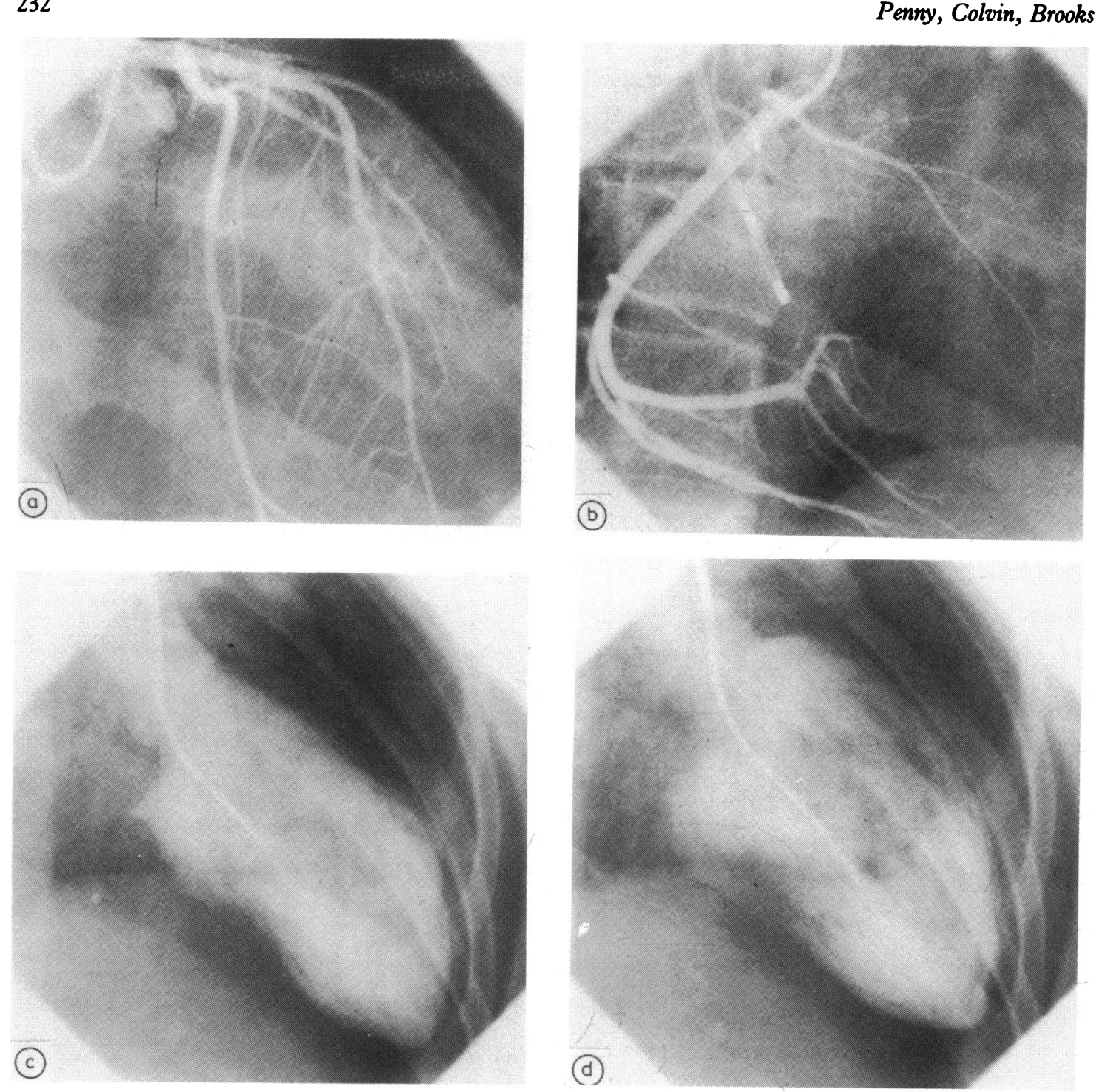

Fig. 2 Coronary arteriogram three months after myocardial infarction. (a) and (b) Coronary arteries are normal. The left ventriculogram in systole (c) and diastole (d) continued to show extensive apical akinesia but the mural thrombus had resolved.

tic methods (Kabi Diagnostica, Stockholm), protein $\mathrm{C}$ by Laurell electrophoresis, and plasminogen by a caseinolytic method. Fibrinolytic activator was measured by placing euglobulin precipitates on unheated fibrin plates and assessing zones of lysis after incubation overnight. Platelet function tests were done in an axiodistogingival aggregometer with standard concentrations of adenosine diphosphate, adrenaline, collagen and ristocetin.
These investigations (Table) showed a prolonged kaolin cephalin time owing to a partial deficiency of factor XII (Hageman factor), which was $27 \%$ of the control concentration. There was no evidence of inhibitory activity, and tests for the lupus inhibitor gave negative results. These findings were confirmed on two subsequent occasions and in an independent laboratory. Platelet aggregation induced by diminished adrenaline and collagen concentrations 
Table Results of tests for haemostasis. These findings were confirmed on two subsequent occasions and in an independent laboratory

\begin{tabular}{|c|c|c|c|}
\hline & Patient & Control & Normal range \\
\hline $\begin{array}{l}\text { Prothrombin ratio } \\
\text { Kaolin cephalin time (s) } \\
\text { Thrombin time (s) } \\
\text { Factor VII (IU/ml) } \\
\text { Factor IX (IU/ml) } \\
\text { Factor XI (\%) } \\
\text { Factor XII (\%) } \\
\text { Prekallikrein (Fletcher) (\%) } \\
\text { Antithrombin III (IU/ml) } \\
\text { Protein C (\%) } \\
\text { Antiplasmin (\%) } \\
\text { Euglobulin lysis zone }\left(\mathrm{mm}^{2}\right)\end{array}$ & $\begin{array}{l}1 \cdot 3 \\
60 \\
10 \\
0 \cdot 9 \\
0 \cdot 6 \\
82 \\
27 \\
64 \\
0 \cdot 91 \\
95 \\
130 \\
87\end{array}$ & $\begin{array}{r}1 \\
42 \\
10\end{array}$ & $\begin{array}{l}110-1 \cdot 3 \\
35-48 \\
10-12 \\
0 \cdot 5-2 \cdot 0 \\
0 \cdot 5-2 \cdot 0 \\
50-200 \\
50-200 \\
50-200 \\
0 \cdot 8-1 \cdot 2 \\
\text { Not established } \\
2-4 \\
60-100\end{array}$ \\
\hline
\end{tabular}

^Equal volume mix of normal and test plasma $42 \mathrm{~s}$.

was compatible with treatment with aspirin.

\section{Discussion}

Our patient showed unequivocal electrocardiographic, cardiac enzyme, and angiographic evidence of acute myocardial infarction. Coronary angiography at three days showed extensive filling defects, which had the appearance of thrombus, in the left coronary circulation. The coronary arteries themselves appeared to be normal, and this was confirmed by repeat angiography three months later.

One previous case report has described this sequence, but the 29 year old man reported on was subsequently found at necropsy, four months after angiography gave "normal" results, to have a $75 \%$ atherosclerotic stenosis at the site of the previous occlusion. ${ }^{5}$ Angiography is highly unlikely to have failed to detect appreciable atheromatous disease in the young woman we have described.

In some cases a prolonged episode of coronary vasospasm may be responsible for myocardial infarction in the absence of coronary atherosclerosis. A thrombotic occlusion could theoretically result from stasis caused by prolonged coronary spasm, and this was recently reported in a case of myocardial infarction that complicated selective coronary angiography. ${ }^{6}$ We have no evidence that spasm played a part in our patient. The hypothesis that spasm of the main left coronary artery caused thrombosis distally in its two branches is, however, attractive. Another remote possibility is that coronary spasm caused extensive infarction and there was subsequent embolisation from the mural thrombus, but this would seem to be a suprising coincidence. We found no evidence of a potential for paradoxical embolism.

The finding of a moderate deficiency of factor XII was unexpected. Factor XII is important not only in the intrinsic coagulation pathway but also in plasminogen dependent fibrinolysis, the kallikrein-kinin system, and complement activation. ${ }^{7}$ The absence of a tendency to bleed abnormally in most cases of factor XII deficiency together with the reported incidence of thromboembolism ${ }^{78}$ suggests that these other reactions may be of clinical importance. Both these reports $^{78}$ include the evidence of reduced fibrinolytic capacity, but this could not be shown in our patient. At least two cases of myocardial infarction in patients with factor XII deficiency have been reported, 910 but both patients were middle aged and probably had coronary artery disease.

In reports of myocardial infarction with subsequently normal coronary arteries the aetiology of the presumed thrombotic occlusion has not been known except when it has complicated coronary angiography and has been caused by a catheter. ${ }^{6}$ An analysis of conventional risk factors has implicated smoking induced thrombosis, ${ }^{11}$ but this has not been an important risk factor in other series, ${ }^{12}$ and, although factor XII deficiency could theoretically be relevant in our patient, the association might be coincidental. Long term follow up over many years may provide further clues, but the present case suggests that we need to extend our concept of classical risk factors in young patients with myocardial infarction and normal coronary arteries.

We thank Dr R Balcon, consultant cardiologist, London Chest Hospital, for permission to report this case; and Dr P B A Kernoff, codirector of the haemostasis unit, Royal Free Hospital, for his help with the assays of prekallikrein, protein C, antiplasmin, and plasminogen.

\section{References}

1 Bertrand ME, Lefebvre M, Laisne CL, Rousseau MF, Carre AG, Lekieffre TP. Coronary arteriography in acute transmural myocardial infarction. Am Heant f 1979; 97: 61-9.

2 Betriu A, Pare JC, Sanz GA, et al. Myocardial infarction with normal coronary arteries: a prospective clinicalangiographic study. Am $\mathcal{F}$ Cardiol 1981; 48: 28-32.

3 Aznar J, Fernandez-Pavón A. Thromboembolic accidents in patients with congenital deficiency of Factor XII. Thrombosis et Diathesis Haemorrhagica 1974; 31: $525-6$.

4 Austen DEG, Rhymes IL. A laboratory manual of blood coagulation. Oxford: Blackwell Scientific Publications, 1975.

5 Lindsay J Jr, Dwyer S, Punja U. Angiographic demonstration of coronary occlusion during spontaneous acute myocardial infarction and subsequent angiographically normal coronary arteries. Am $\mathcal{F}$ Cardiol 1983; 51: 1227-8.

6 Vincent GM, Anderson JL, Marshall HW. Coronary 
spasm producing coronary thrombosis and myocardial infarction. N Engl f Med 1983; 309: 220-3.

7 McPherson RA. Thromboembolism in Hageman trait. Am $\mathcal{J}$ Clin Pathol 1977; 68: 420-3.

8 Dyerberg J, Stoffersen E. Recurrent thrombosis in a patient with Factor XII deficiency. Acta Haematol (Basel) 1980; 63: 278-82.

9 Glueck HI, Roehll W. Myocardial infarction in a patient with a Hageman (factor XII) defect. Ann Intern Med 1966; 64: 390-6.
10 Hoak JC, Swanson LW, Warner ED, Connor WE. Myocardial infarction associated with severe factor XII deficiency. Lancet 1966; ii: 884-6.

11 McKenna WJ, Chew CYC, Oakley CM. Myocardial infarction with normal coronary angiogram. Possible mechanism of smoking risk in coronary artery disease. $\mathrm{Br}$ Heart f 1980; 43: 493-8.

12 Khan AH, Haywood LJ. Myocardial infarction in nine patients with radiologically patent coronary arteries. $N$ Engl f Med 1974; 291: 427-31. 